\title{
On the Distribution of Signal Phase in Body Area Networks
}

\author{
Simon L. Cotton, Ugo S. Dias, William G. Scanlon, and Michel D. Yacoub
}

\begin{abstract}
In this letter, we investigate the distribution of the phase component of the complex received signal observed in practical experiments using body area networks. Two phase distributions, the recently proposed $\kappa-\mu$ and $\eta-\mu$ probability densities, which together encompass the most widely used fading models, namely Semi-Gaussian, Rayleigh, Hoyt, Rice, and Nakagami- $m$, have been compared with measurement data. The $\kappa-\mu$ distribution has been found to provide the best fit over a range of on-body links, while the user was mobile. The experiments were carried out in two dissimilar indoor environments at opposite ends of the multipath spectrum. It has also been found that the uniform phase distribution has not arisen in anyone of the experiments.
\end{abstract}

Index Terms $-\kappa-\mu$ phase distribution, on-body propagation.

\section{INTRODUCTION}

I $\mathrm{N}$ the last few years, significant improvements to the understanding of the communications process between wireless devices situated on the human body have been made [1]-[5]. Investigations carried out to characterise the signal propagation through on-body channels found in body area networks (BANs) have revealed the following. The envelope, correlation, and crossing rate statistics may follow those of the fading channels as widely known in conventional wireless communications systems. These, nevertheless, depend upon user state, environment, type of antenna, and on-body location [1]-[4]. However, to the best of the authors' knowledge, no study has appeared in the literature that investigates the distribution of phase in BANs. Phase distribution for fading signals is indeed a challenging topic and has been raising the attention of researchers for many years [6]-[8]. In particular, an interesting discussion in this matter concerns the appropriateness of the use of uniform phase distribution in the sum of random vectors, which distinguishes a fading signal [6]. The phase statistics have a direct impact on the performance of $M$-phase signaling over fading channels using diversity [9]. Hence, the results presented here will be of interest and benefit to those evaluating and developing communications techniques for use in BANs. The aim of this Letter is two fold: (i) to report the result of phase measurements carried in BANs, to the best of the authors' knowledge, an unprecedented work in the literature; and (ii) to give an account of the phase probability density function (PDF) that yields the best fit to the measured data. Two phase fading models, namely $\kappa-\mu[10]$ and $\eta-\mu[11]$, are used that together encompass the most widely

Manuscript received April 23, 2010. The associate editor coordinating the review of this letter and approving it for publication was G. Karagiannidis.

S. L. Cotton and W. G. Scanlon are with the School of Electronics, Electrical Engineering and Computer Science, Queen's University, Belfast, BT3 9DT, UK (e-mail: \{simon.cotton,w.scanlon\}@qub.ac.uk).

U. S. Dias was with the Wireless Technology Laboratory, University of Campinas, Brazil. He is now with the Department of Electrical Engineering, University of Brasilia, 70910-900, DF, Brazil (e-mail: udias@unb.br).

M. D. Yacoub is with the Wireless Technology Laboratory, Department of Communications, University of Campinas, 13083-970, SP, Brazil (e-mail: michel@wisstek.org).

This work was partly supported by the UK Engineering and Physical Sciences Research Council - EPSRC (EP/H044191/1).

Digital Object Identifier 10.1109/LCOMM.2010.08.100673 deployed fading scenarios, namely Semi-Gaussian, Rayleigh, Hoyt, Rice, and Nakagami- $m$. It is anticipated that the $\eta-\mu$ phase PDF has been consistently outperformed by the $\kappa-\mu$ phase PDF and that the uniform phase PDF has not arisen in anyone of these experiments.

\section{II. $\kappa-\mu$ PHASE Distribution}

The $\kappa-\mu$ distribution has recently been proposed as a fading model which can represent the small-scale signal variation of a fading signal under line-of-sight (LOS) (or existence of dominant signal components) and multipath clustering conditions [12]. It is a general distribution, which contains a number of other distributions as special cases. For $\mu=1$, the $\kappa$ - $\mu$ model reduces to Rice, in which case the $\kappa$ parameter coincides with the Rice $k$ factor. For $\kappa \rightarrow 0$, the $\kappa$ - $\mu$ model reduces to Nakagami- $m$, in which case the parameter $\mu$ coincides with the Nakagami $m$ parameter. From Rice as well as from Nakagami- $m$, Rayleigh can be obtained. In the $\kappa-\mu$ model, the parameter $\mu$ is related to the number of multipath clusters in the environment and $\kappa$ is defined as the ratio between the total power of the dominant components and the total power of the scattered waves [12]. For a $\kappa$ - $\mu$ fading signal with envelope $R$ and phase $\Theta$, and a scaled envelope $\mathrm{P}=2 \mu \sqrt{\kappa(1+\kappa)} R / \hat{r}$, with $\hat{r}=\sqrt{E\left(R^{2}\right)}$, where $E(\cdot)$ signifies the expectation operation, the scaled-envelope-phase joint $\operatorname{PDF} f_{\mathrm{P}, \Theta}(\rho, \theta)$ is given as in (1), for the general case (dominant component in both in-phase and quadrature signals) [10]. Simpler forms of (1) can be found in [10] for $\phi= \pm n \pi$ (dominant component only in the in-phase signal) and for $\phi= \pm(2 n+1) \pi / 2$ (dominant component only in the quadrature signal). In (1), $\phi=\arg (p+j q)$ is a phase shift parameter, $p$ and $q$ representing the amplitudes of the dominant components of inphase and quadrature signals, respectively. The phase PDF is obtained as $f_{\Theta}(\theta)=\int_{0}^{\infty} f_{\mathrm{P}, \Theta}(\rho, \theta) d \rho$, for which, at present, no closed-form solution is available, although its computation is indeed straightforward. It is noteworthy that the particular cases comprised by the $\kappa-\mu$ envelope PDF, namely Rice and Nakagami- $m$, are also encompassed in an exact manner by the $\kappa-\mu$ phase PDF [10].

\section{EXPERIMENTS}

Two separate measurement environments, namely an anechoic chamber and a reverberation chamber, were chosen. These environments are considered to be representative of the minimum (anechoic) and maximum (reverberation) environmental multipath conditions likely to be experienced by everyday BAN users. To facilitate a separate study of the signal phase variation due to body movements, the mechanical stirrers in the reverberation chamber were disabled. Seven on-body receiver node locations, representative of a BAN, were also selected. These were head, front chest, waist, knee, ankle, wrist, and elbow all situated on the right side of the user's body. The transmitter was located at the user's left waist ensuring that all on-body links were in Non-LOS. The reader is referred to Fig. 1. The antennas used in this study were compact $(5 \mathrm{~mm}$ height) higher mode microstrip 


$$
f_{\mathrm{P}, \Theta}(\rho, \theta)=\frac{\rho^{\mu+1}|\sin (2 \theta)|^{\frac{\mu}{2}} \exp \left(-\rho^{2} / 4 \kappa \mu+\rho \cos (\theta-\phi)\right) I_{\frac{\mu}{2}-1}(\rho|\cos \theta \cos \phi|) I_{\frac{\mu}{2}-1}(\rho|\sin \theta \sin \phi|)}{8(2 \kappa \mu)^{\mu}|\sin (2 \phi)|^{\frac{\mu}{2}-1} \cosh (\rho \cos \theta \cos \phi) \cosh (\rho \sin \theta \sin \phi) \exp (\kappa \mu)}
$$

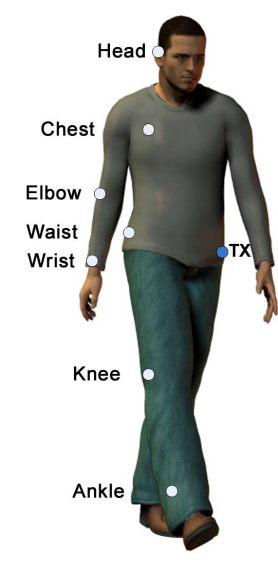

Fig. 1. Wireless nodes on the human body forming a WBAN.

patch antennas. The test subject was an adult male of height $1.82 \mathrm{~m}$ and mass $90 \mathrm{~kg}$. In the experiments, carried out at $2.45 \mathrm{GHz}$, the antennas were mounted so that the radiating patch element was parallel to the body surface. In addition, they were connected to a vector network analyser (Rhode \& Schwarz ZVB-8 VNA) using calibrated low-loss coaxial cables. The VNA was configured to record measurements of $S_{21}$ at $5 \mathrm{~ms}$ intervals for 30 seconds. For all measurements, the user performed two trials of a walking on the spot motion at a set location in both environments. Each of the individual, complex received envelopes analysed in this study consisted of 6000 samples, giving a cumulative total of 168000 samples over both experiments.

\section{REsults}

The data were collected, processed, and tested against the theoretical phase PDFs of $\eta-\mu$ [11] and $\kappa-\mu$ [10] models. As well known, these encompass the most widely used fading models, namely Semi-Gaussian, Rayleigh, Hoyt, Rice, and Nakagami- $m$. Although developed for Non-LOS channels, in these experiments the $\eta-\mu$ phase distribution was consistently outperformed by the $\kappa-\mu$ phase distribution according to model ranking performed using the Akaike information criterion [1]. This suggests that even though no direct LOS existed between the bodyworn antenna locations, dominant signal components, possibly formed by the on-body creeping wave [5] or reflected and scattered from the body surface, were present. Interestingly, the uniform phase distribution showed in no one of the experiments performed here. In addition, it became clear from the results that the multimodal phase behaviour, due to multipath clustering, as predicted by the $\eta-\mu$ and $\kappa-\mu$ models, constantly showed. Table I presents a summary of the parameter estimates for the $\kappa-\mu$ phase distribution. These were obtained using a generalised nonlinear least squares routine written in MATLAB to perform minimization on data sets of 3000 samples per link. Table I also shows the Kullback-Liebler divergence $^{1}\left(D_{K-L}\right)$ between theoretical and practical phase

\footnotetext{
${ }^{1}$ The Kullback-Leibler divergence $D_{K-L}$ between PDFs $f_{X}(x)$ and $f_{Y}(x)$ is defined as $D_{K-L}=\int_{-\infty}^{\infty} f_{X}(x) \ln \frac{f_{X}(x)}{f_{Y}(x)} d x$, where, in general, $f_{X}(x)$ and $f_{Y}(x)$ are respectively the true and test PDFs. In case these are zeromean, unity variance and zero-mean, $\sigma^{2}$ variance Gaussian PDFs, then $D_{K-L}=0.5\left(\sigma^{-2}-1\right)+\ln \sigma$.
}

PDFs. As a term of comparison, Table I depicts the standard deviation $\sigma$ of a zero-mean, $\sigma^{2}$ variance Gaussian PDF distant $D_{K-L}$ from a zero-mean, unity-variance Gaussian PDF. In the low multipath conditions of the anechoic chamber, the antenna positions closest to the transmitter, i.e., the chest and waist experienced the highest $\kappa$ values. Therefore it would be expected to see the phase distribution being dominated by the phase of strongest components from each of the contributing signal clusters arriving at the receiver. Furthermore, it is also clear from Table I that for the majority of antenna locations, the magnitude of the $\kappa$ parameter reduced, in some instances quite considerably (chest and waist), when the user moved between the anechoic and reverberation chambers. This provides solid evidence that when a BAN is expected to operate in a highly multipath environment, signal contributions arriving from an off-body direction become an important part of the channel model and cannot be ignored.

In the plots, for clarity we chose to depict the practical measurements confronted only with the best fit $\kappa-\mu$ theoretical curves. (As noted earlier, the $\eta-\mu$ model was consistently outperformed by the $\kappa-\mu$ one.) As sample examples, the results for the chest, head, knee, and waist locations are shown in Figs. 2, 3, 4, and 5, respectively. The excellent agreement of the theoretical $\kappa-\mu$ phase PDF with the measured data can be seen for all on-body links in both environments (including those not shown here). Note how the $\kappa$ - $\mu$ phase PDF tends to keep track of the various modes of the true PDF. An important characteristic of these figures is the shape of the estimated phase densities. Under anechoic conditions, the spread of the measured phase tended to increase for antenna locations farther from the transmitter. This suggests that contributing signal components may travel along paths of dissimilar lengths and therefore arrive from increasingly different directions. In the reverberation chamber, scattered contributions will also arrive from nearby interfering objects and the metallic shielded structure. Note that the densities for the reverberation condition take a much broader (flattened) shape than those of the anechoic ones, although they are far from the uniform, as initially assumed in many fading channel models.

\section{CONCLuSions}

It has been shown that the phase distribution in BANs is greatly affected by the transmission path length and the multipath conditions of the local environment. Using two recently proposed distributions, namely $\eta-\mu$ and $\kappa-\mu$, which jointly encompass the well-known fading models found in the literature, the $\kappa-\mu$ distribution was found to best model the distribution of phase in BANs. A range of parameter estimates for this distribution have been reported so that the phase characteristics measured in this study may be readily reproduced.

\section{REFERENCES}

[1] S. L. Cotton, G. A. Conway, and W. G. Scanlon, "A time-domain approach to the analysis and modeling of on-body propagation characteristics using synchronized measurements at $2.45 \mathrm{GHz}$," IEEE Trans. Antennas Propag., vol. 57, no. 4, pp. 943-955, Apr. 2009. 
TABLE I

ESTIMATED PARAMETERS FOR $\kappa-\mu$ PHASE DISTRIBUTION FOR ALL EXPERIMENTAL TRIALS.

\begin{tabular}{|c|c|c|c|c|c|c|c|c|c|c|}
\hline Position & \multicolumn{4}{|c|}{ Anechoic } & \multicolumn{5}{|c|}{ Reverberation } \\
\hline & $\hat{\kappa}$ & $\hat{\mu}$ & $\hat{\phi}$ & $D_{K-L}$ & $\sigma$ & $\hat{\kappa}$ & $\hat{\mu}$ & $\hat{\phi}$ & $D_{K-L}$ & $\sigma$ \\
\hline Ankle & 0.44 & 0.96 & -0.14 & 0.1583 & 0.7037 & 0.17 & 1.15 & 0.18 & 0.1279 & 0.7264 \\
\hline Chest & 10.87 & 0.77 & -0.30 & 0.0708 & 0.7831 & 0.34 & 1.05 & 0.39 & 0.1790 & 0.6901 \\
\hline Elbow & 0.34 & 1.08 & 0.37 & 0.1749 & 0.6927 & 0.55 & 1.10 & 0.06 & 0.1157 & 0.7367 \\
\hline Head & 0.44 & 1.17 & 0.47 & 0.1664 & 0.6982 & 0.22 & 1.17 & -0.11 & 0.1874 & 0.6849 \\
\hline Knee & 1.21 & 1.03 & 0.35 & 0.0778 & 0.7747 & 0.60 & 1.30 & 0.13 & 0.1168 & 0.7357 \\
\hline Waist & 0.17 & 5.00 & -0.83 & 0.0773 & 0.7753 & 0.40 & 1.20 & -0.31 & 0.2088 & 0.6725 \\
\hline Wrist & 0.28 & 1.18 & 0.25 & 0.1696 & 0.6961 & 0.31 & 1.15 & 0.00 & 0.1542 & 0.7065 \\
\hline
\end{tabular}

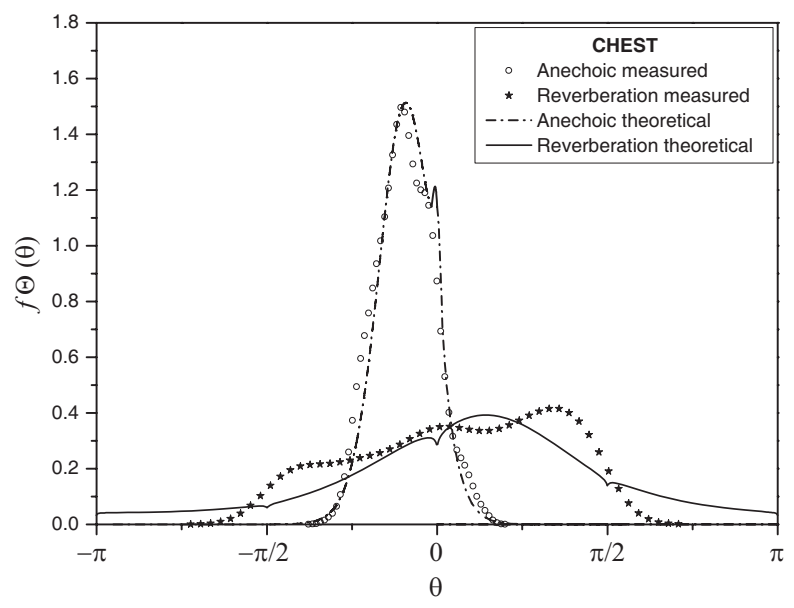

Fig. 2. Measured and theoretical phase PDFs for chest positioned antennas while the user was mobile in anechoic and reverberation chambers.

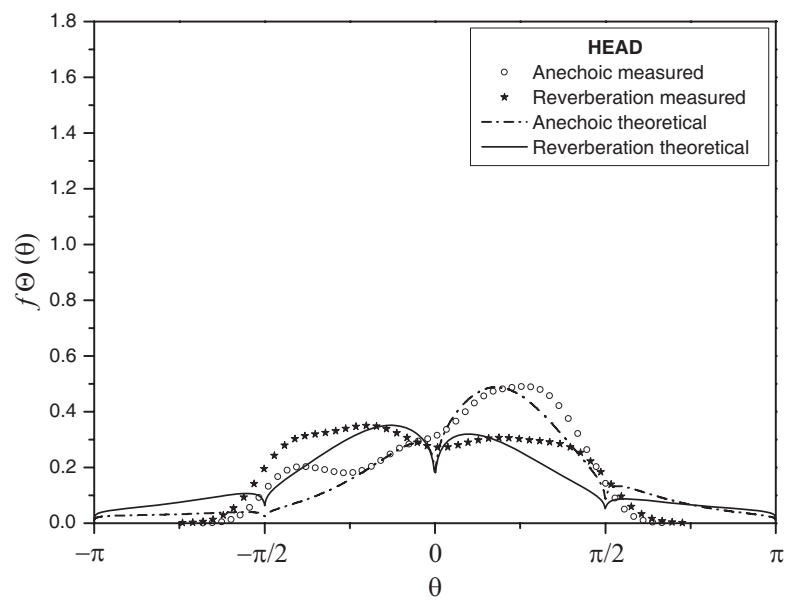

Fig. 3. Measured and theoretical phase PDFs for head positioned antennas while the user was mobile in anechoic and reverberation chambers.

[2] D. B. Smith, J. Zhang, L. W. Hanlen, D. Miniutti, D. Rodda, and B. Gilbert, "Temporal correlation of dynamic on-body area radio channel," IET Electron. Lett., vol. 45, no. 24, pp. 1212-1213, Nov. 2009.

[3] I. Khan, Y. I. Nechayev, and P. S. Hall, "On-body diversity channel characterization," accepted, IEEE Trans. Antennas Propag., 2010.

[4] S. L. Cotton and W. G. Scanlon, "High-order statistics for $\kappa-\mu$ distribution," IET Electron. Lett., vol. 43, no. 22, Oct. 2007.

[5] G. A. Conway, S. L. Cotton, and W. G. Scanlon, "An antennas and propagation approach to improving physical layer performance in wireless body area networks," IEEE J. Sel. Areas Commun., vol. 27, no. 1, pp. 27-36, Jan. 2009.

[6] E. Jakeman and P. N. Pusey, "A model for non-Rayleigh sea echo," IEEE Trans. Antennas Propag., vol. 24, no. 6, pp. 806-814, Nov. 1976.

[7] G. T. F. de Abreu, "CTH16-4: accurate simulation of piecewise continuous arbitrary Nakagami- $m$ phasor processes," in Proc. IEEE Globecom, San Francisco, CA, USA, Nov. 2006, pp. 1-6.

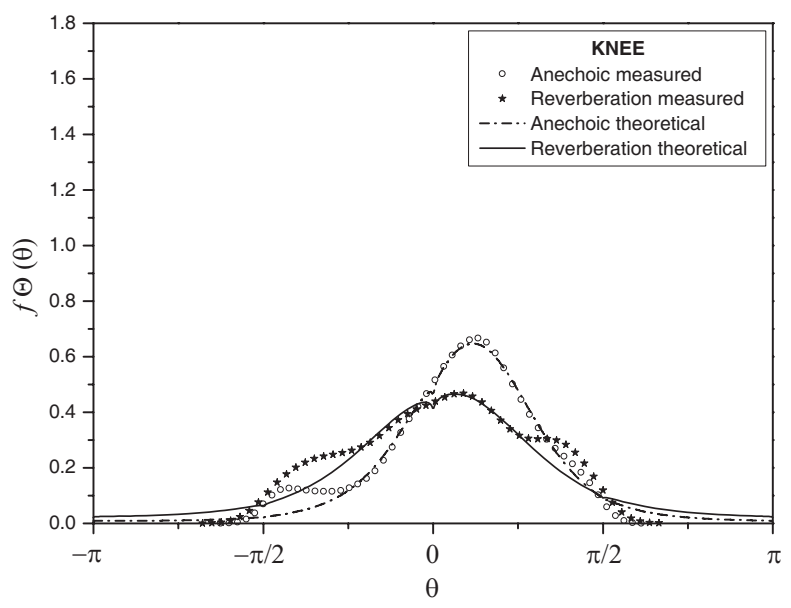

Fig. 4. Measured and theoretical phase PDFs for knee positioned antennas while the user was mobile in anechoic and reverberation chambers.

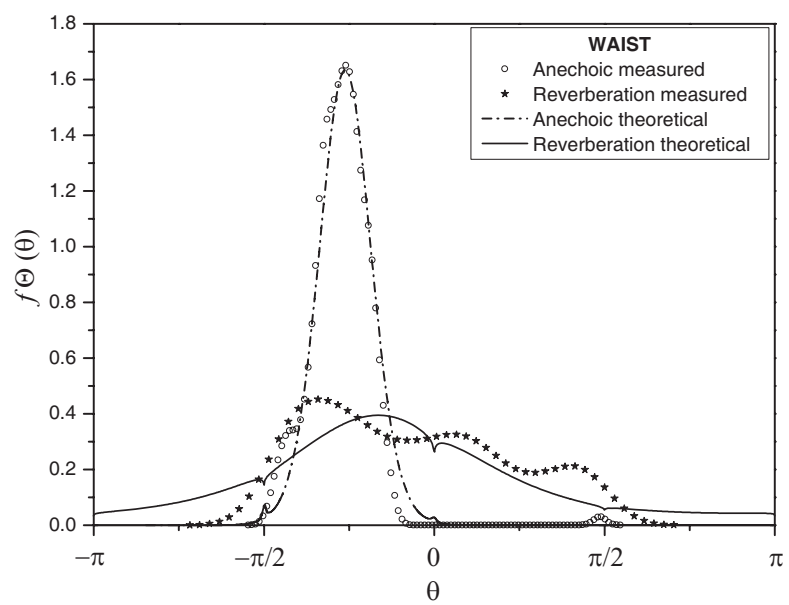

Fig. 5. Measured and theoretical phase PDFs for waist positioned antennas while the user was mobile in anechoic and reverberation chambers.

[8] Y. Ma and D. Zhang, "A method for simulating complex Nakagami fading time series with nonuniform phase and prescribed autocorrelation characteristics," IEEE Trans. Veh. Technol., vol. 59, no. 1, pp. 29-35, Jan. 2010.

[9] J. G. Proakis, Digital Communications, 4th edition. New York: McGraw-Hill, 2001.

[10] U. S. Dias and M. D. Yacoub, "The $\kappa-\mu$ phase-envelope joint distribution," IEEE Trans. Commun., vol. 58, no. 1, pp. 40-45, Jan. 2010.

[11] D. B. da Costa and M. D. Yacoub, "The $\eta-\mu$ joint phase-envelope distribution," IEEE Antennas Wireless Propag. Lett., vol. 6, pp. 195198, 2007.

[12] M. D. Yacoub, "The $\kappa-\mu$ and the $\eta-\mu$ distribution," IEEE Antennas Propag. Mag., vol. 49, no. 1, pp. 68-81, Feb. 2007. 